\title{
1 Une histoire de la surveillance de la qualité de l'eau des 2 milieux naturels en France
}

3 A history of water quality monitoring in natural environment in

4 France

5

Y. ZAITER ${ }^{1 *} ;$ F. DESTANDAU $^{1}$

7

8

\section{Résumé}

Les réseaux de surveillance de l'eau jouent un rôle important dans la collecte d'information sur la qualité et la quantité de l'eau. Pour l'aspect quantitatif, les premiers réseaux de surveillance datent de 1840. La surveillance qualitative de la ressource en eau a, quant à elle, débuté avec la loi sur l'eau de 1964. Ces réseaux ont, par la suite, connu une évolution au fil des lois et Directives européennes sur l'eau. Dans cet article, nous retraçons, dans une première partie, I'histoire de ces réseaux de surveillance en France en distinguant trois périodes : les premiers réseaux découlant de la loi sur l'eau de 1964, les évolutions résultant des Directives européennes des années 70 à 90, et enfin les réseaux de surveillance depuis la Directive-cadre sur l'eau de 2000. Dans une seconde partie, nous nous intéressons en particulier au bassin Rhin-Meuse, en présentant les différents réseaux existants : la déclinaison des réseaux nationaux exposés précédemment, mais également les réseaux locaux spécifiques.

\section{Mots Clés}

Gestion de l'eau ; Qualité de l'eau ; Réseaux de surveillance ; Lois et Directives sur l'eau ; Approche historique

\begin{abstract}
Water monitoring networks play an important role in collecting information on water quality and quantity. For the quantitative aspect, the first monitoring networks date back to 1840. The qualitative monitoring of water resources only began with the 1964 water law. These networks have subsequently evolved in the line with laws and European Directives on water. In this article, we first retrace the history of these monitoring networks in France by distinguishing three periods: the first networks resulting from the 1964 water law, the changes resulting from the European Directives of the 1970s to 1990s, and finally the monitoring networks since the Water Framework Directive of 2000. In the second part, we are particularly interested in the Rhine-Meuse catchment, presenting the different existing networks: the adaptation of the national networks described above, but also the specific local networks.
\end{abstract}

\section{Key Words}

Water management; Water quality; Monitoring networks; Water laws and Directives; Historical approach.

\footnotetext{
${ }^{1}$ ENGEES, UMR GESTE MA-8101, Strasbourg, France. E-mail address: youssef.zaiter@engees.unistra.fr
} 


\section{Introduction}

A l'époque napoléonienne la législation sur l'eau ne portait que sur le droit de propriété des eaux. Le code civil fixait la propriété et l'usage de l'eau des différents cours d'eau. L'article 714 précisait notamment qu'un bien qui n'a pas de propriétaire est la propriété du public. Ainsi, tous les individus avaient le droit d'utiliser ces biens publics tels que l'air ou l'eau.

Avec la révolution industrielle de la fin du XIX ${ }^{\text {ème }}$ siècle, les volumes et la nature des usages de l'eau ont évolué. Une grande quantité d'eau était désormais consommée pour la production. Pour prendre en compte ce changement, la loi du 8 avril 1898 a été votée pour réorganiser l'utilisation de l'eau [BLUM et al., 2010]. Cette loi visait à identifier les besoins et à garantir l'accès à la ressource pour chaque acteur: agriculteurs, industriels, particuliers. Les eaux étaient réglementées par des zones dédiées à des usages spécifiques. À cette époque, la principale préoccupation était de réglementer l'utilisation de l'eau et le législateur n'accordait aucune attention à la qualité de l'eau [BOULEAU, 2014].

Le premier réseau de surveillance est, ainsi, quantitatif et date de 1840. II s'agissait de mesurer la quantité d'eau disponible dans la Nappe de l'Albien à Paris [ROUX, 2006]. Ces réseaux se sont ensuite développés dans plusieurs régions françaises toujours pour des eaux souterraines. L'objectif était essentiellement de connaître la quantité d'eau disponible pour alimenter les usines et les locomotives à vapeur.

Concernant la surveillance de la qualité, le Ministère en charge de la santé organise et pilote l'acquisition de données sur les eaux brutes et distribuées dès 1960, dans le cadre réglementaire du contrôle sanitaire des eaux destinées à l'alimentation en eau potable. Ce réseau est géré au niveau départemental par les Directions Départementales des Affaires Sanitaires et Sociales (DDASS) [BLUM et al., 2010]. Mais c'est surtout à partir de la loi sur l'eau de 1964 que la surveillance de la qualité des milieux aquatiques commence à s'organiser sur l'ensemble du territoire.

Dans cet article nous traiterons, dans une première partie, de l'évolution de la surveillance au niveau national au gré des nouvelles lois et Directives européennes. Puis, dans une seconde partie, des réseaux de surveillance complémentaires au niveau local, en prenant pour exemple le bassin RhinMeuse.

\section{L'évolution des réseaux de surveillance en France}

L'évolution historique de la surveillance de la qualité de l'eau au niveau national sera présentée en trois périodes. Chacune de ces sections sera organisée selon le même découpage en sous-sections : Contexte législatif, surveillance des eaux de surface, surveillance des eaux souterraines, données de stockage, et indicateur de qualité. 


\subsection{La loi sur l'eau de 1964 et le premier réseau de surveillance}

\subsubsection{Contexte législatif}

L'après-guerre a été caractérisé par une croissance démographique et une industrialisation rapides. Cette situation a perturbé le cycle de l'eau et a entraîné une augmentation de la pollution aquatique. Toutefois la réglementation, héritée de la troisième république (1870-1940), ne se préoccupait pas de la qualité de l'eau [BOULEAU, 2014]. Le terme «pollution » lui-même, n'était utilisé que par deux groupes de pression principaux : les hygiénistes pour définir une eau qui ne pouvait pas être utilisée pour l'approvisionnement domestique, et les pêcheurs pour dénoncer l'impact nocif sur les poissons [BOULEAU, 2014]. En 1959, un débat s'ouvre au niveau de l'Etat au sujet des problèmes de pollution de l'eau. Le Commissariat général au plan créé la commission de l'eau afin d'établir une loi générale sur l'eau pour réorganiser la gestion de l'eau. Cette loi est votée le 16 décembre 1964. Elle pose «les bases de ce qui est devenu l'école française de gestion de l'eau » [LESAGE, 2013], avec une gestion à l'échelle des grands bassins hydrographiques avec la création des comités de bassin, et l'application du principe pollueur-payeur par le biais des Agences de l'eau.

Dans son article 3, la loi sur l'eau de 1964 instaure également l'obligation de surveiller la pollution des eaux de surface par la création de l'Inventaire National du degré de Pollution des eaux superficielles (INP).

\subsubsection{Surveillance des eaux de surface : Inventaire national du degré de pollution des eaux superficielles}

Créé par le décret du 10 janvier 1969, I'Inventaire National du degré de Pollution des eaux superficielles (INP) avait pour mission d'offrir un premier aperçu de la qualité des cours d'eau. Le réseau était géré par les Agences de l'eau.

Les paramètres mesurés étaient au nombre de 66. II s'agissait de paramètres physico-chimiques (température, potentiel en hydrogène, oxygène, potassium, chlorures, nitrates, matières en suspension, etc.), de micropolluants minéraux, de métaux (fer, manganèse, zinc, etc.), de paramètres environnementaux (présence de boue, mousse de détergents, etc.), organiques (microbiologie), ou autres micropolluants (radioactivité, pesticides, etc.) [PETIT, 2010].

L'article 2 du décret de 1969 précise que le Ministre, chargé du plan et de l'aménagement du territoire, fixe, pour chaque bassin, par arrêté ministériel et sur recommandation du comité de bassin, le nombre et la localisation des stations de surveillance, la fréquence et les dates du début du prélèvement.

La fréquence d'échantillonnage a été fixée à une fois tous les cinq ans. Trois campagnes d'échantillonnage ont eu lieu : 1971, 1976 et 1981.

Les stations de surveillance étaient situées sur les grandes rivières, en aval des sources de pollution et dans des endroits où la population et l'activité industrielle étaient importantes. II s'agissait donc 


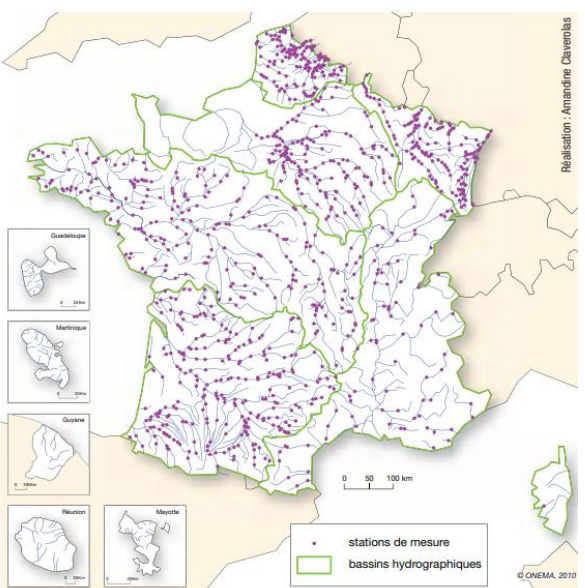

Deux des trois campagnes étant marquées par des périodes de sécheresse (1976 et 1981), le 111 dispositif est annualisé à partir de 1981 pour obtenir une image plus complète et moins aléatoire de la qualité des cours d'eau. [PETIT, 2010 ; LARONDE et PETIT, 2010]

\subsubsection{Surveillance des eaux souterraines}

115 Sur la demande des Agences de l'eau et des Conseils régionaux, désireux de suivre la pollution des nappes par les nitrates d'origine agricole, la qualité des eaux souterraines commença à être suivie en

117 1970. Cependant, en 1970 seule une soixantaine de "qualito-mètres » sont présents exclusivement 118 dans le bassin Rhin-Meuse et sur l'île de la Réunion [BLUM et al. 2013] (Figure 2).

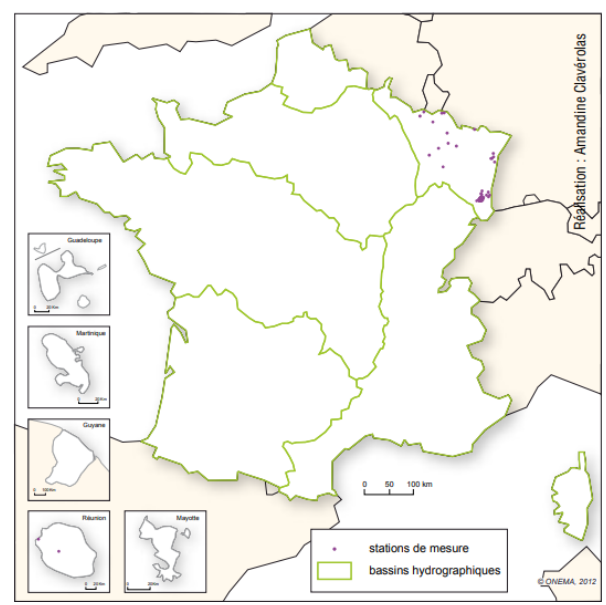

Figure 2. Stations de mesure de la qualité des eaux souterraines en 1970 [BLUM et al., 2013] 
122 Durant les années 1970 et 1980 une vingtaine de réseaux, dits "patrimoniaux ", sont créés en 123 France, dans le but de suivre l'évolution de la qualité des nappes et proposer des actions pour les 124 protéger. 48 paramètres sont recherchés, principalement physico-chimiques.

\subsubsection{Stockage et diffusion des données}

127 Avec la création des réseaux de surveillance, l'information sur l'état des eaux est devenue abondante.

128 La question du stockage et de la diffusion de ces données s'est ainsi posée. Jusqu'en 1992, chaque 129 producteur de données (services de l'état, organismes d'étude et de recherche, etc.) produisait sa 130 propre banque de données sans règle commune pour s'assurer de leur homogénéité et comparabilité 131 [LARONDE et PETIT, 2010].

\subsubsection{Indicateur de qualité}

134 Le premier outil d'évaluation ayant servi à transformer les données en indicateurs de qualité fut la 135 grille de 1971 [LARONDE et PETIT, 2010].

136 Cinq niveaux de qualité (Excellente, Bonne, Passable, Médiocre, Très mauvaise), associés à des 137 valeurs seuils de chaque paramètre, permettaient d'évaluer l'aptitude de l'eau à répondre à des 138 usages du plus au moins contraignants en termes de qualité (Tableau 1)

Tableau 1 Grille de 1971 [LARONDE et PETIT, 2010]

\begin{tabular}{|l|c|c|c|c|c|}
\hline Classe & 1A & 1B & 2 & 3 & HC \\
\hline Qualité & Excellente & Bonne & Passable & Médiocre & $\begin{array}{c}\text { Très } \\
\text { mauvaise }\end{array}$ \\
\hline $\begin{array}{l}\text { Qualité } \\
\text { minimale } \\
\text { requise pour : }\end{array}$ & Baignade & $\begin{array}{c}\text { Vie normale des } \\
\text { poissons }\end{array}$ & $\begin{array}{l}\text { Industrie } \\
\text { Irrigation }\end{array}$ & Navigation & \\
\hline $\begin{array}{l}\text { Traitement de } \\
\text { potabilisation }\end{array}$ & \multicolumn{2}{|c|}{ Simple } & Difficile & $\begin{array}{c}\text { Quasi } \\
\text { impossible }\end{array}$ \\
\hline
\end{tabular}

141

142 Les paramètres pris en compte pour l'analyse de la qualité de l'eau étaient les polluants présents dans

143 l'assiette des redevances des Agences de l'eau à l'époque: matières organiques, azotées et 144 phosphorées. 


\subsubsection{Contexte législatif}

149 Au cours des années 1970 à 1990, deux vagues de Directives européennes concernant la qualité de

150 l'eau ont été adoptées. Les Directives européennes découlent des Programmes d'Action 151 Environnementale (PAE) qui, depuis 1973, fournissent le cadre général pour la politique 152 environnementale de l'Union européenne (voir Figure 3).

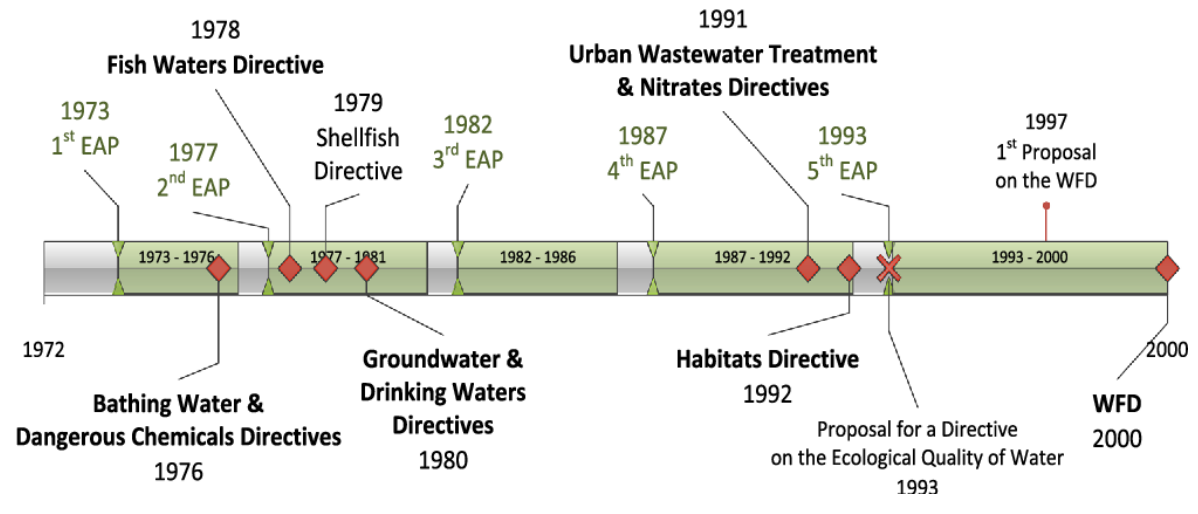
Urban Wastewater Treatment \& Nitrates Directives
rban Wastewater Treatm

Figure 3. PAE et Directives européennes [LANZ et SCHEUER, 2001]

157 La première vague de législation a eu lieu de 1975 à 1980 (deux premiers PAE). Cette vague a donné lieu à un certain nombre de Directives concernant les normes de qualité environnementale ou l'établissement de valeurs limites d'émission pour des usages spécifiques de l'eau: Directive 75/440/CEE relative aux eaux de surface (1975) ; Directive 76/464/CEE concernant les substances dangereuses (1976) ; Directive 78/659/CEE relative aux eaux piscicoles (1978) ; Directive 79/923/CEE relative aux eaux conchylicoles (1979); Directive 80/86/CEE relative aux eaux souterraines (1980). avec deux Directives mettant en place des obligations de moyens pour limiter des rejets spécifiques : Directive 91/676/CEE relative aux nitrates (1991) ; Directive 91/271/CEE relative au traitement des eaux urbaines résiduaires (1991).

167 Pour intégrer ces nouvelles exigences européennes, la France adopte une nouvelle loi sur l'eau en 1992.

169 La loi sur l'eau de 1992 définit la ressource en eau comme un patrimoine national commun (article 1)

170 et précise l'importance de la préservation de la ressource en eau (article 2). Pour définir les objectifs 171 quantitatifs et qualitatifs et les méthodes à mettre en œuvre, la loi introduit les SDAGE (Schéma 172 Directeur d'Aménagement et de Gestion d'Eau) pour chaque district hydrographique, et les SAGE 173 (Schéma d'Aménagement et de Gestion d'Eau), pour les sous bassins. Elle crée également les 174 Directions Régionales de l'Environnement (DIREN) chargées de coordonner la mise en place de la 
politique environnementale au niveau régional (Direction Régionale de l'Aménagement et de 176 Logement (DREAL) depuis 2011). Toutefois, la surveillance de la qualité de l'eau n'est pas 177 mentionnée dans la loi.

\subsubsection{Surveillance des eaux de surface : Le Réseau National de Bassin}

En 1987, suite aux Directives européennes des années 1975 à 1980 et de l'hétérogénéité des réseaux de surveillance existants, le gouvernement a lancé une proposition de réorganisation du réseau de surveillance. L'objectif était d'avoir un réseau national cohérent et uniforme intégrant les rivières et petits ruisseaux. La mise en place du réseau s'est faite selon un protocole national entre le ministère de l'environnement et les Agences de l'eau. Le protocole a été convenu entre les six Agences de l'eau afin de maintenir la cohérence des mesures. Le Réseau National de Bassin (RNB) a été créé et a remplacé l'INP. L'établissement du RNB s'est fait selon deux principes fondamentaux : la représentativité spatio-temporelle de l'information sur la qualité de l'eau, et la cohérence des mesures au niveau national [LARONDE et PETIT, 2010].

Les objectifs du RNB étaient de : connaître l'état général de la ressource en eau, connaître les principaux polluants qui entraînent la dégradation de la qualité de l'eau, donner des informations sur la qualité de l'eau après la mise en œuvre de la politique européenne et nationale de l'eau, évaluer l'impact des mesures mises en œuvre pour restaurer la qualité de l'eau, fournir à tous les gestionnaires de l'eau des renseignements sur la qualité de l'eau.

Le RNB était, ainsi, structuré en trois réseaux :

i) Réseaux patrimoniaux : pour évaluer l'état général de l'eau et évaluer les tendances.

ii) Réseaux d'utilisation : pour définir les différentes usages de l'eau en fonction de leur qualité.

iii) Réseaux d'impact : pour évaluer l'impact des activités ou industries polluantes. minimal de stations de surveillance par bassin (250 stations de surveillance), l'emplacement des stations de surveillance, les paramètres à mesurer (physico-chimie classique, microbiologie, micropolluants et hydrobiologie) et la fréquence des prélèvements :

- 6 ou 12 mesures par an pour le physico-chimique et les micropolluants ;

- 9 mesures par an pour la microbiologie. 
211 Parallèlement au RNB, la plupart des Agences de l'eau ont mis en œuvre des réseaux 212 complémentaires de bassin (RCB) et ont incité les collectivités à mettre en place des réseaux locaux 213 pour mieux couvrir certaines zones du territoire [PETIT, 2010].

\subsubsection{Surveillance des eaux souterraines}

Afin d'atteindre les objectifs fixés par la loi sur l'eau de 1992, le financement des Agences de l'eau et des collectivités locales s'est accru au début des années 1990. Ainsi, plusieurs réseaux de surveillance de la qualité des eaux souterraines gérés par des collectivités locales ont été créés et le nombre de qualito-mètres a augmenté passant à 368 en 1993, soit cinq fois plus qu'en 1970 [BLUM et al., 2013].

En 1999, s'est posé la question de l'harmonisation de ces nouveaux réseaux. Les Agences de l'eau fixent ainsi une méthodologie commune en termes de densité minimale de stations de surveillance, de fréquence des prélèvements, de paramètres chimiques à suivre. II en résulte le Réseau National de connaissances des Eaux Souterraines (RNES), et plus spécifiquement pour la qualité le RNES-Q.

Le RNES-Q a entraîné une augmentation du nombre de stations de surveillance de la qualité de 368 en 1993 à 2149 en 2001 (Figure 5). Le nombre de paramètres a, quant à lui, doublé.

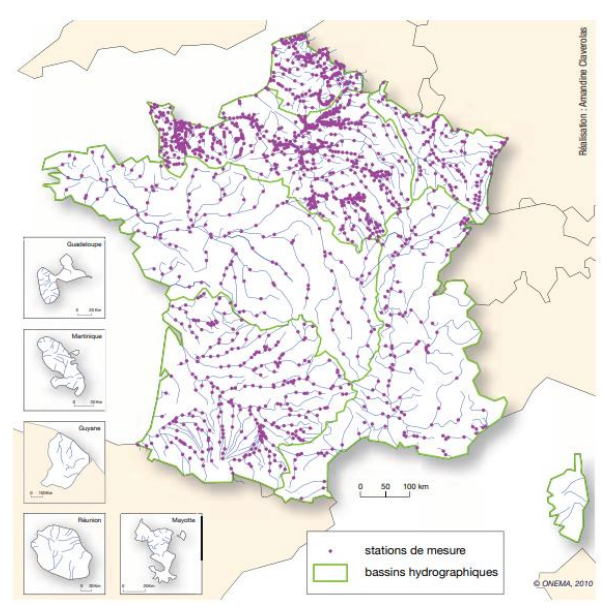

Figure 4. Stations de mesure RNB en 1987 [PETIT, 2010] 

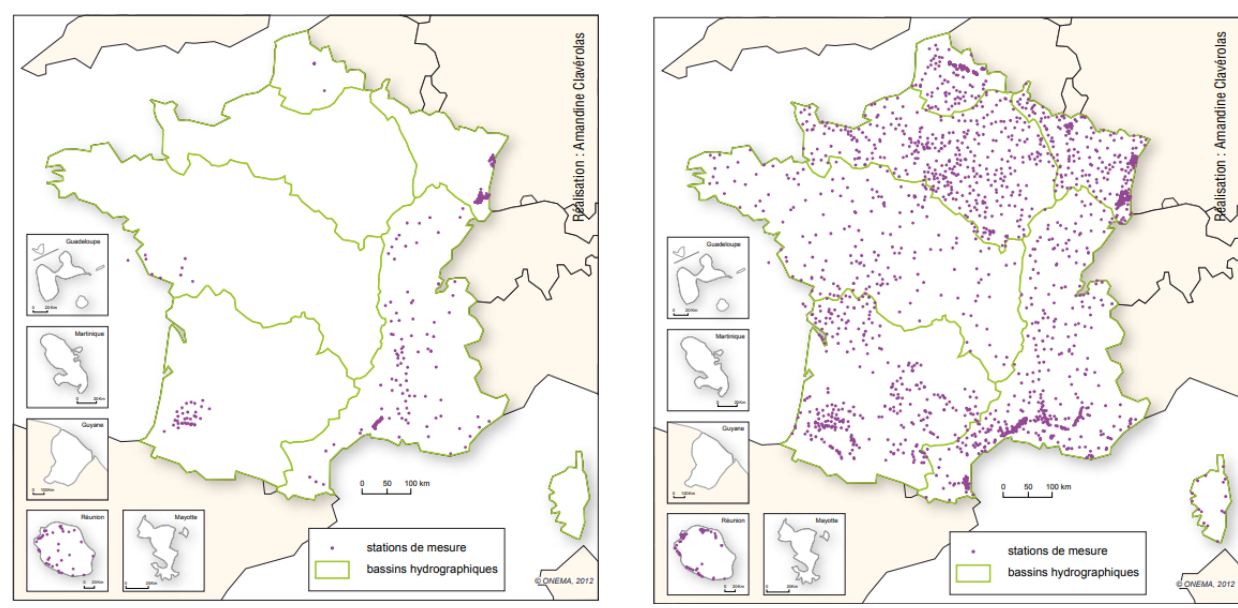

Figure 5. Stations de mesure de la qualité des eaux souterraines en 1993 (gauche) et 2001 (droite) [BLUM et al., 2013]

Suite aux Directives européennes de 1991 relatives aux nitrates et au traitement des eaux urbaines résiduaires (1991), le nombre de stations de surveillance a augmenté afin d'avoir une vision plus claire de l'état des eaux et des impacts environnementaux existants comme l'eutrophisation. Le nombre de paramètres mesurés a augmenté de 130 en 1987, à 758 en 2006. Les paramètres étaient liés à l'état physico-chimique de l'eau. De nouvelles analyses concernant les micropolluants pharmaceutiques, hydro-biologiques et organiques sont apparues en 2001. La fréquence d'échantillonnage a également augmenté suite à la réorganisation des paramètres visés pour répondre aux normes de qualité imposées par les Directives européennes entre 1980 et 1990.

Ainsi, la Directive européenne du 12 décembre 1991, dénommée Directive Nitrate (Directive 91/676/CEEE), prévoit la réalisation d'une campagne de surveillance des teneurs en nitrate dans les eaux douces au moins tous les 4 ans [LALLOUETTE et PETIT, 2012]. Un réseau de points de surveillance, appelé réseau nitrate a été progressivement constitué.

Les stations qui composent le réseau nitrate sont choisies par les bassins pour rendre compte de la concentration des eaux en nitrates d'origine agricole sur l'ensemble du territoire [LALLOUETTE et PETIT, 2012]. Le nombre des stations de mesure pour la première campagne étaient 3104 stations de mesure en France (1939 pour les eaux souterraines et 1165 pour les eaux superficielles). Ce chiffre a progressivement augmenté durant les années pour arriver à 5937 points de mesure pour la campagne de 2018-2019 [MINISTERE DE LA TRANSITION ECOLOGIQUE ET SOLIDAIRE, 2018].

Les paramètres suivis par ce réseau sont: le phosphore total, les ortho-phosphates, la demande biochimique en oxygène à 5 jours (DBO5), l'oxygène dissous, les nitrites et les nitrates, et la chlorophylle-a. 


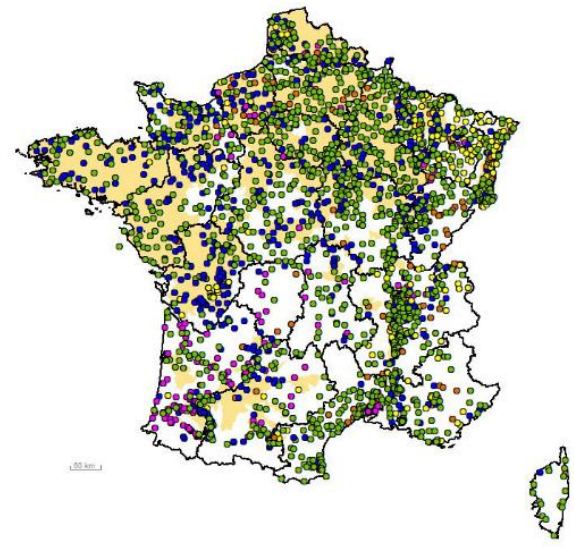

Figure 6 : Localisation des stations de mesures nitrate en eaux souterraines [LALLOUETTE et PETIT. 2012]
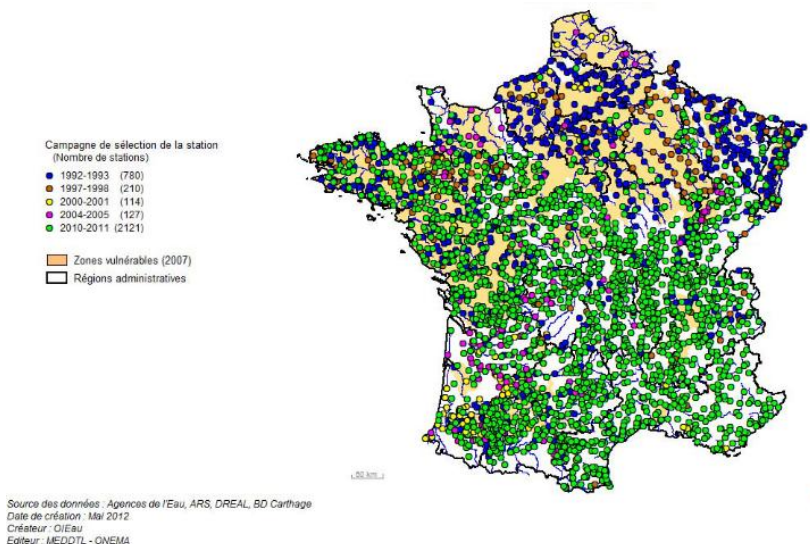

Figure 7 : Localisation des stations de mesures nitrate en eaux superficielle [LALLOUETTE et PETIT. 2012]

En plus des réseaux de surveillance ci-dessus, une veille de la qualité des eaux est également effectuée dans un but sanitaire. Ce dispositif de veille et de sécurité sanitaire est encadré par le code de la santé publique et qui vient en application de la Directive européenne 98/83/CE relative à la qualité des eaux destinées à la consommation humaine [AGENCE REGIONALE DE SANTE, 2012]. Cette Directive précise les exigences de la qualité auxquelles doit satisfaire l'ensemble des valeurs mesurées pour chaque paramètre. Ces exigences de qualité sont fondées sur les évaluations menées par l'organisation mondiale de la santé.

Cette surveillance se fait en France depuis les années 1998 à travers les Agences Régionales de Santé (ARS) qui organisent le contrôle sanitaire réglementaire [www.grand-est.ars.sante.fr]. Ce réseau ARS comprend:

- Des analyses: prélèvements réalisés au niveau des ressources, des installations de traitement et au robinet du consommateur ;

- Des inspections/contrôles : vérification de la conformité des installations et des mesures de sécurité sanitaires mises en œuvre. 
Au niveau national, les ARS effectuent une surveillance annuelle sur 33200 captages en France 274 [AGENCE REGIONALE DE SANTE, 2017]. La répartition géographique de ces captages n'est pas 275 homogène sur le territoire français, leur nombre variant selon les départements (Figure 8).

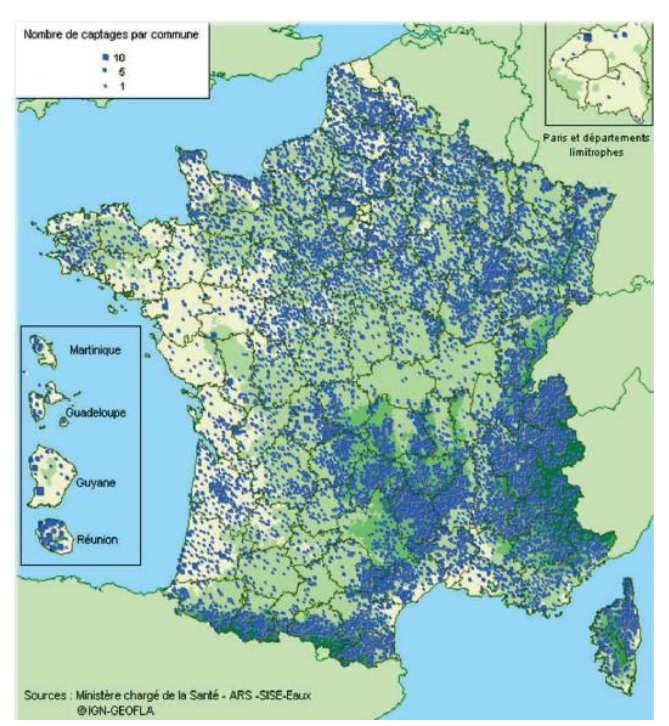

Figure 8 Répartition des captages utilisés pour la production d'eau potable en France ; [AGENCE REGIONALE DE SANTE, 2012]

\subsubsection{Stockage et diffusion des données}

Suite à la loi sur l'eau de 1992, les producteurs de données sur l'eau ont signé un protocole afin de coordonner et harmoniser la conservation et la diffusion des données par le biais du Réseau National des Données sur l'Eau (RNDE).

Le RNDE est ainsi une organisation partenariale entre les principaux producteurs de données publiques relatives à l'eau: Ministère de l'environnement, Agences de l'eau, BRGM (Bureau de Recherche Géologiques et Minières), Conseil Supérieur de la pêche, EDF (Electricité de France), Ifremer (Institut Français de Recherche pour l'Exploitation de la MER), Météo-France, Institut Français pour l'Environnement, Office International de l'Eau. En tant qu'opérateur technique du RNDE, l'Office international de l'eau a créé en 1994 la Banque Nationale des Données sur l'Eau (BNDE) pour assurer une centralisation des données en vue d'une diffusion à l'échelle nationale [LARONDE et PETIT, 2010].

Pour inciter à la normalisation de ces données, le SANDRE (Service d'Administration Nationale des Données et Référentiels sur l'Eau) a été créé en 1993. (1) 


\subsubsection{Indicateur de qualité}

300 La loi sur l'eau de 1992 a conduit à revoir la grille d'évaluation de la qualité des milieux aquatiques pour intégrer les nouveaux paramètres mesurés. Le nouvel outil d'évaluation: SEQ (Système d'Evaluation de la Qualité), intégraient trois composantes :

- SEQ-Eau : Évaluer la qualité physique et chimique du plan d'eau ;

Le SEQ-Eau évalue la qualité de l'eau selon cinq niveaux de qualité : Très bonne, Bonne, Passable, Mauvaise, Très mauvaise (Figure 9).

INDICES
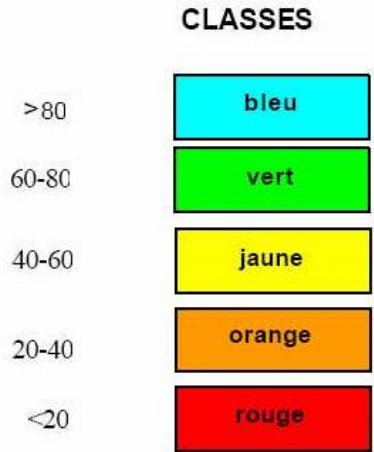

QUALITE

Très bonne

Bonne

Passable

Mauvaise

Très mauvaise

Figure 9 Evaluation de la qualité de l'eau par classe dans le système SEQ-Eau²

L'aptitude à satisfaire des usages par l'homme (production d'eau potable, loisirs et sports aquatiques, irrigation, abreuvage des animaux, aquaculture) et le développement harmonieux de la flore et de la faune aquatique (aptitude à la biologie) sont évalués, pour chaque paramètre de chacune des altérations, à partir de grilles spécifiques à chaque usage et à la fonction biologie.

\subsection{Directive cadre sur l'eau et réseaux actuels}

\subsubsection{Contexte législatif}

Le 23 octobre 2000, la Commission européenne a adopté la Directive-cadre sur l'eau (Directive 2000/60/CE). Contrairement aux autres Directives européennes, la Directive-cadre sur l'eau (DCE) fixe des objectifs de résultat à atteindre dans des délais déterminés (cycles 2015, 2021, 2027). Elle reprend le principe français de gestion décentralisée par bassin versant, et impose une méthode commune à l'ensemble des Etats membres de l'Union européenne (Figure 10).

\footnotetext{
${ }^{2}$ Source : http://hmf.enseeiht.fr/travaux/CD0506/bei/bei_ere/7/html/b1/projet/SEQ.htm
} 


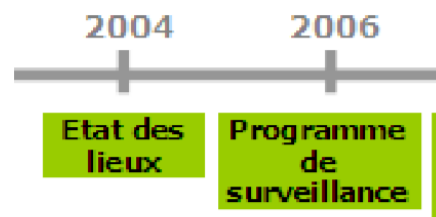

Figure 10. Calendrier pour le premier cycle DCE [LARONDE et PETIT, 2010]

L'article 8 de la DCE précise que les Etats membres veillent à la mise en place de programmes de surveillance afin d'établir une vue d'ensemble cohérente ${ }^{3}$ et complète de l'état des eaux dans chaque district hydrographique.

La loi LEMA (Loi sur l'Eau et les Milieux Aquatiques) n²006-1772, transpose la DCE dans une loi française au 30 décembre 2006.

\subsubsection{Surveillance des eaux de surface}

Cinq réseaux de surveillance des eaux de surface sont requis par la DCE (Agence de l'eau RhinMeuse $^{4}$ ) : le réseau du contrôle de surveillance (RCS), le réseau du contrôle opérationnel (RCO), le réseau du contrôle d'enquête, le réseau du contrôle additionnel et le réseau de référence. Les réseaux de surveillance ont été construits sur la base de la circulaire du 13 juillet 2006 [LARONDE et PETIT, 2010], et de la circulaire de 11 avril 2007. 
stations de surveillance ont été créées en fonction de cet objectif. Selon la circulaire du 13 juillet 2006 relative à la mise en place de réseaux de surveillance de la qualité des eaux de surface, le réseau est composé de 1500 stations de surveillance réparties à part égale entre districts de bassin en France métropolitaine. II existe 250 stations de surveillance par bassin.

Cette circulaire indique également la méthode pour localiser les stations de surveillance qui est basée sur deux approches.

La première est la prise en compte de la taille des cours d'eau :

- Très petit : 1 station de surveillance pour chaque tronçon de 175 à 250 km ;

- Petit : 1 station de surveillance pour chaque tronçon de 150 à $200 \mathrm{Km}$;

- Moyenne : 1 station de surveillance pour chaque tronçon de 40 à $70 \mathrm{Km}$; et

- Grande : 1 station de surveillance par masse d'eau ${ }^{5}$.

La deuxième approche est une approche de préoccupation statistique basée sur l'examen de la variabilité observée des résultats, par paramètre mesuré, dans les différentes classes de taille.

Le réseau est géré par trois acteurs différents : l'AFB (Agence Française pour la Biodiversité), la DREAL et les Agences de l'eau. Les trois parties définissent les paramètres biologiques et physicochimiques à mesurer.

La fréquence d'échantillonnage est de 6 à 12 fois par an pour les paramètres chimiques, alors qu'elle est de 1 à 2 fois par an pour les paramètres biologiques.

Le réseau de contrôle opérationnel (RCO) est un réseau de surveillance temporaire qui surveille uniquement les masses d'eau qui risquent de ne pas atteindre le bon état selon la circulaire DCE 2005/12 du 28 juillet 2005 relative à la définition du bon état. Pour ces masses d'eau, un report d'échéance ou un objectif moins strict est fixé dans le cadre du schéma directeur d'aménagement et de gestion des eaux (SDAGE) [Circulaire 13 juillet 2006]. Ainsi, le contrôle opérationnel a pour but de suivre l'évolution de la qualité de la masse d'eau suite aux actions mises en place dans le cadre des programmes de mesures. Le réseau mesure les mêmes paramètres que le RCS mais avec un nombre réduit de stations de surveillance. Une fois que la masse d'eau atteint l'état environnemental et écologique requis, ce réseau arrête la surveillance.

Le réseau de contrôle d'enquête est un réseau de surveillance temporaire qui vise à identifier les sources de pollution responsables du mauvais état de la masse d'eau.

Le réseau de contrôle additionnel est un réseau qui accentue la surveillance sur certaines zones protégées, comme les zones Natura 2000 et les captages d'eau de surface fournissant en moyenne plus de $100 \mathrm{~m}^{3} /$ jour.

Le réseau de référence est un réseau dont le but est de qualifier une eau non perturbée par les activités anthropiques, afin de mesurer l'impact de l'activité humaine sur les autres masses d'eau. Ce

\footnotetext{
${ }^{5}$ La masse d'eau est le découpage élémentaire des milieux aquatiques destinée à être l'unité d'évaluation de la DCE. II peut s'agir d'un lac, d'un réservoir, d'un petit bassin versant, d'une partie de rivière, de fleuve, de canal...etc...
} 
réseau a été créé en France par la circulaire DCE 2007/11 du 11 avril 2007 relative au protocole de prélèvement et traitement des échantillons des invertébrés pour la mise en œuvre du programme de surveillance des cours d'eau.

\subsubsection{Surveillance des eaux souterraines}

A l'instar des eaux de surface, les eaux souterraines se découpent désormais en masses d'eau, qui deviennent les unités d'évaluation de leur état. Le bon état dépend de la quantité et de l'état chimique (qualité).

Concernant la surveillance de l'état chimique, il existe deux réseaux de surveillance : le RCS et le RCO, déjà évoqués pour les eaux de surface. Le RCS remplace le RNES-Q en 2007, et le RCO, qui vise les masses susceptibles de ne pas atteindre le bon état chimique, est mis en place en 2008. [BLUM et al. 2013]. Leur répartition est désormais assez homogène sur l'ensemble du territoire. Le nombre de paramètres à suivre passe de 1200 à 1400 .

\subsubsection{Stockage et diffusion des données}

398 Pour répondre aux exigences de la DCE sur la nécessité d'un système d'information permettant de connaître la qualité des milieux aquatiques et d'identifier les causes de leur dégradation, une modernisation du stockage des données était nécessaire.

401 Ainsi, une réflexion débuta en 2002 pour simplifier les modalités de financement, définir les principes 402 d'organisation des réseaux, et établir un bilan-diagnostic des réseaux de données sur tout le territoire.

403 II en résulta la définition d'un Schéma Directeur des Données sur l'Eau dans chaque bassin (SDDE) 404 qui aboutit à la signature d'un nouveau protocole par les partenaires du RNDE donnant naissance au 
Système d'Information sur l'Eau (SIE). La mise en place et la coordination technique du SIE est confiée en 2006 à l'Onema.

Le SIE est conçu pour répondre aux besoins des parties prenantes en matière d'information environnementale publique dans le domaine de l'eau [CHERY et al., 2008]. Différents types de portails sont concernés :

- Un portail parent multithématiques : eaufrance ;

- Des portails nationaux thématiques: plan d'eau, cours d'eau, eaux littorales, eaux souterraines, hydrologie, données piscicoles; à terme, s'ajouteront les données sur l'assainissement, les prélèvements, les usages et les pressions, les données économiques ;

Parmi les portails cités ci-dessus, s'ajoute le portail ADES (Accès aux Données sur les Eaux Souterraines) qui est le portail national pour les eaux souterraines [CHERY et al., 2008]. Le portail ADES rassemble sur un site public des données quantitatives et qualitatives relatives aux eaux souterraines. Les objectifs sont les suivants :

- Constituer un outil de collecte et de conservation des données sur les eaux souterraines ;

Cette base de données permet de connaître et de localiser les réseaux et les stations de mesure et d'accéder aux résultats qualitatifs et quantitatifs. Les résultats présents dans cette base de données sont donnés par point de mesure et par bassin versant.

\subsubsection{Indicateur de qualité}

Après la transposition de la DCE en droit français, le système d'évaluation de la qualité de l'eau est apparu obsolète. Pour évaluer les données fournies par les nouveaux réseaux de surveillance, un nouvel outil d'évaluation a été créé : le Système d'Evaluation de l'Etat des Eaux (SEEE).

L'évaluation de la qualité de l'eau est définie par l'arrêté ministériel du 25 janvier 2010, qui définit son état écologique et chimique. Les paramètres concernés sont au nombre de 895. Cependant, le nombre de paramètres varie en fonction du bassin et des stations de surveillance.

438 Les paramètres reçoivent une note pour l'état écologique et chimique. Cinq catégories sont définies pour l'état écologique : Très bon, Bon, Moyen, Médiocre, Mauvais. Pour l'état chimique, la qualité est 
masse d'eau est considérée en Bon état si l'état écologique est Bon ou Très bon, et l'état chimique Bon (Figure 13).

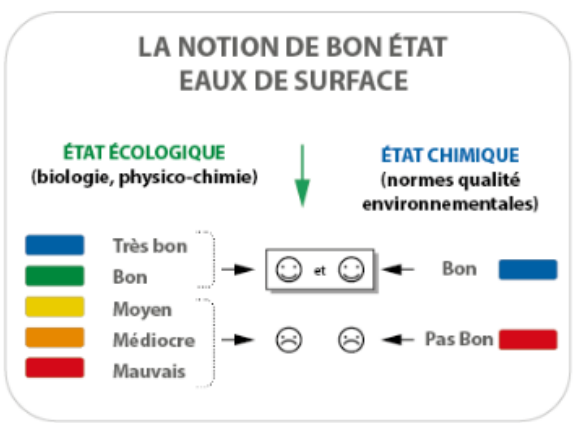

Figure 13. Système d'Evaluation de l'Etat des Eaux ${ }^{6}$

\section{Les réseaux locaux : application au bassin Rhin-Meuse}

\subsection{Les réseaux nationaux dans le bassin Rhin-Meuse}

- Inventaire national du degré de pollution des eaux superficielles (INP) : Historiquement, le premier réseau de surveillance de la qualité de l'eau pour le bassin Rhin-Meuse était l'INP, dont la première campagne de mesure date de 1971. Comme partout en France, ce réseau a été créé à la suite de la loi sur l'eau de 1964 qui mentionnait l'obligation de surveiller la qualité de l'eau. Les stations étaient localisées de manière à identifier les points de noirs de pollution. Dans le bassin Rhin-Meuse, ce réseau ne comptait qu'une dizaine de stations de surveillance.

- Réseau national de bassin (RNB) : Dans les années 80, le RNB remplace l'INP dans le bassin RhinMeuse. L'objectif était de mettre en place un réseau de suivi stable. En 2006, le RNB comprend 230 stations de surveillance dans le bassin Rhin-Meuse, dont certaines stations de l'INP.

- Réseau Nitrate : Le réseau de surveillance nitrate est composé de 276 stations de mesure sur le bassin Rhin Meuse [AGENCE DE L'EAU RHIN-MEUSE, 2016 a,b]. Nous pouvons noter que la localisation de ces stations n'est pas spécifique à ce réseau. D'autres paramètres peuvent être mesurés dans le cadre d'autres réseaux.

- Réseau de surveillance ARS (Agence Régionale de Santé) : Dans le bassin Rhin-Meuse, I'ARS Grand-Est est responsable du contrôle sanitaire des eaux distribuées. Elle effectue une surveillance annuelle de la qualité de l'eau potable. Elle a réalisé en 2017 plus de 33000 prélèvement d'échantillons d'eau pour analyses. Ce prélèvement d'eau est fait auprès de 3855 captages d'eau brute $^{7}$ (avant le traitement de l'eau potable).

- Réseaux obligatoires DCE : Dans le bassin Rhin-Meuse, le Réseau de contrôle de surveillance (RCS) a été créé en 2007 comme partout en France. II comprend 107 stations de surveillance des eaux de surface et 196 stations de surveillance des eaux souterraines [AGENCE DE L'EAU RHIN-

\footnotetext{
${ }_{7}^{6} \mathrm{http}: / /$ sage-dol.fr/sage-4-20-30-contexte-legal-et-reglementaire-du-sage.html

${ }^{7}$ www.grand-est.ars.sante.fr/la-qualite-de-leau-potable
} 
MEUSE, 2016 $6^{a, b}$. L'Agence de l'eau a choisi de reprendre un maximum de stations de surveillance du RNB. Parmi les 107 stations des eaux superficielles du RCS, 85 étaient déjà dans le réseau RNB.

Le Réseau de contrôle opérationnel (RCO) comprend, en 2007, 301 stations de surveillance. Le choix des paramètres surveillés diffèrent d'une station à l'autre, les paramètres déclassants n'étant pas les mêmes. Le RCO est mis en œuvre pour $75 \%$ des masses d'eau du bassin Rhin-Meuse avec un système de rotation qui vise à surveiller les masses d'eau en mauvais état chacune leur tour.

Le réseau de contrôles additionnels se divise en deux sous-réseaux dans le bassin Rhin-Meuse. Le premier est le réseau qui surveille la qualité de l'eau des zones de captage pour produire de l'eau potable. Les contrôles additionnels sur les points de captage en eau de surface sont inclus dans les points de contrôles sanitaires. Ces sites sont listés dans l'arrêté de bassin. Ils sont au nombre de 18 dans le bassin. Le deuxième est un réseau qui vise à protéger les zones d'habitats et de protection des espèces : zones spéciales de conservation (ZSC) ou zones de protection spéciale (ZPS). Le nombre de stations de surveillance est de 153 pour le bassin.

Le réseau de surveillance de référence comprend dans le bassin Rhin-Meuse 29 stations. Ne trouvant pas de masses d'eau non affectées par l'activité humaine, l'Agence de l'eau Rhin-Meuse a choisi les masses d'eau les moins affectées par l'activité anthropique. La fréquence d'échantillonnage est d'une fois tous les deux mois.

A notre connaissance, il n'existe pas de réseau d'enquête sur le bassin Rhin-Meuse.

\subsection{Les réseaux complémentaires au niveau du bassin Rhin-Meuse}

Au-delà des réseaux obligatoires, à savoir les réseaux nationaux mis en place par des décrets nationaux, chaque bassin peut mettre en place des réseaux dits complémentaires, officialisés par arrêté de bassin (par le préfet de bassin). Ces réseaux sont : le réseau de surveillance des tendances à long terme (RESALTT), le réseau de surveillance des pesticides et le réseau de captage prioritaire.

Dans le bassin Rhin-Meuse, le réseau de surveillance des tendances à long terme (RESALTT), reprend les stations du RNB qui n'ont pas été gardées dans le RCS. L'Agence de l'eau Rhin-Meuse souhaitait, ainsi, obtenir des informations supplémentaires sur l'état des eaux. La poursuite de la surveillance sur ces stations permet, en outre, de disposer de longues chroniques de données qui représentent un enjeu fort de connaissance et de suivi des évolutions à long terme [AGENCE DE L'EAU RHIN-MEUSE, 2016 $6^{\mathrm{a}, \mathrm{b}}$ ]. 161 stations de surveillance constituent le réseau RESALTT sur le bassin Rhin-Meuse. Parmi ces 161 points, 132 sont communs aux réseaux DCE ou Directive Nitrates.

501 Le deuxième réseau complémentaire est le réseau de surveillance des pesticides, mis en œuvre en

5022000 , et comprenant 130 stations de surveillance. L'objectif du réseau, qui suit 450 paramètres, est de 503 surveiller spécifiquement la pollution d'origine agricole. 
Le troisième réseau complémentaire est le réseau de captage prioritaire vis-à-vis des pollutions diffuses agricoles. C'est un réseau de suivi de la qualité des eaux souterraines. II surveille la qualité de 132 captages prioritaires qui ont été identifiés dans le cadre de l'article 27 de la loi grenelle 1 [AGENCE DE L'EAU RHIN-MEUSE, 2016 a,b]. Ce réseau doit permettre de mettre en place des actions visant à réduire les pollutions liées à l'utilisation de fertilisants et de pesticides.

\subsection{Les réseaux complémentaires au niveau local}

\subsubsection{La surveillance de la qualité de la nappe d'Alsace}

512 La surveillance de la qualité de l'eau souterraine est assurée par l'Agence de l'eau Rhin-Meuse.

513 Toutefois, l'Association pour la Protection de la Nappe Phréatique de la Plaine d'Alsace (APRONA)

514 effectue une surveillance complémentaire pour la nappe d'Alsace. L'APRONA est une association

515 créée le 28 mars 1995 à l'initiative du Conseil régional d'Alsace, de l'Agence de l'eau Rhin-Meuse et 516 des Conseils généraux de la région Alsace. Elle est en charge de la gestion et du suivi quantitatif et 517 qualitatif des eaux souterraines en Alsace. Plus précisément, son rôle est d'effectuer des missions 518 d'expertise pour améliorer la connaissance de la nappe d'Alsace, des aquifères du Sundgau et des 519 interactions liées aux activités de surface pouvant faire peser des risques sur la ressource 520 (www.aprona.net).

521 La surveillance de la qualité des eaux souterraines est effectuée environ tous les 6 ans depuis 1991 522 dans le cadre du projet transfrontalier ERMES (Figure 14 pour illustration).

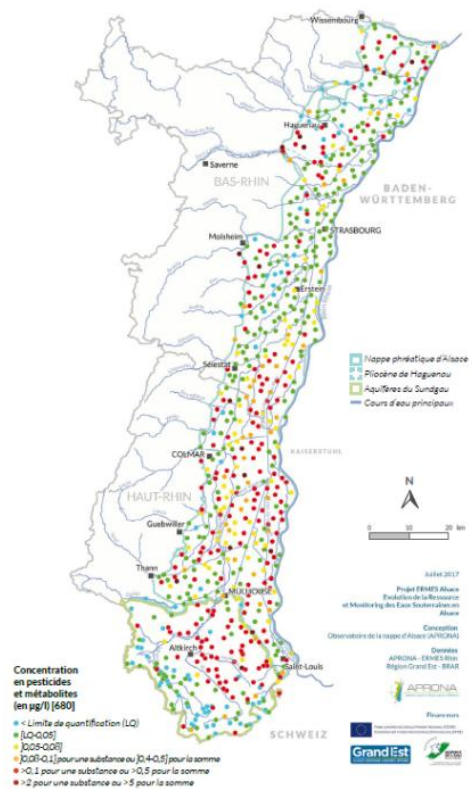


Les principaux acteurs du projet sont français (Aprona, Agence de l'eau Rhin-Meuse, Région GrandEst), allemand (LUBW, HLNUG, RheinlandPfalz) et suisse (Ministère de l'Environnement et de

529 l'Energie, Bâle Landschaft).

530 En 2016, le réseau comprend 1540 stations de surveillance à l'échelle transfrontalière et 825 stations 531 de surveillance en Alsace ${ }^{8}$. Le réseau de surveillance mesure 400 paramètres divisés en deux 532 catégories : les paramètres historiques (nitrate, chlorure, composés organiques halogénés volatils 533 etc.) et les paramètres émergents (composés polyfluorés, substances pharmaceutiques, adjuvants 534 alimentaires etc.).

\subsubsection{Le réseau de surveillance continue du Rhin}

Le $1^{\text {er }}$ novembre 1986, un incendie majeur s'est produit dans un bâtiment de stockage de produits chimiques de l'usine Sandoz à Bâle, en Suisse. Il en a découlé le déversement de trente tonnes de pesticides dans le Rhin. Cela s'est traduit par de graves dommages subis par une grande partie du biotope fluvial [DIEHEL et al., 2005 ; VAN DIJK et al.,1995 ; GIGER, 2009]. Des dommages ont été observés jusqu'à $500 \mathrm{~km}$ à l'aval de l'accident: pollution atmosphérique dans la région de Bâle, répercutions sur l'écosystème du Rhin, et impact sur l'approvisionnement en eau potable.

En réponse à cet accident, la Convention Internationale pour la Protection du Rhin a mis en place en 1987 un Programme d'Action pour le Rhin (PAR). [BROER, 1991]. Ce réseau se compose de 9 stations d'alerte (Figure 15).

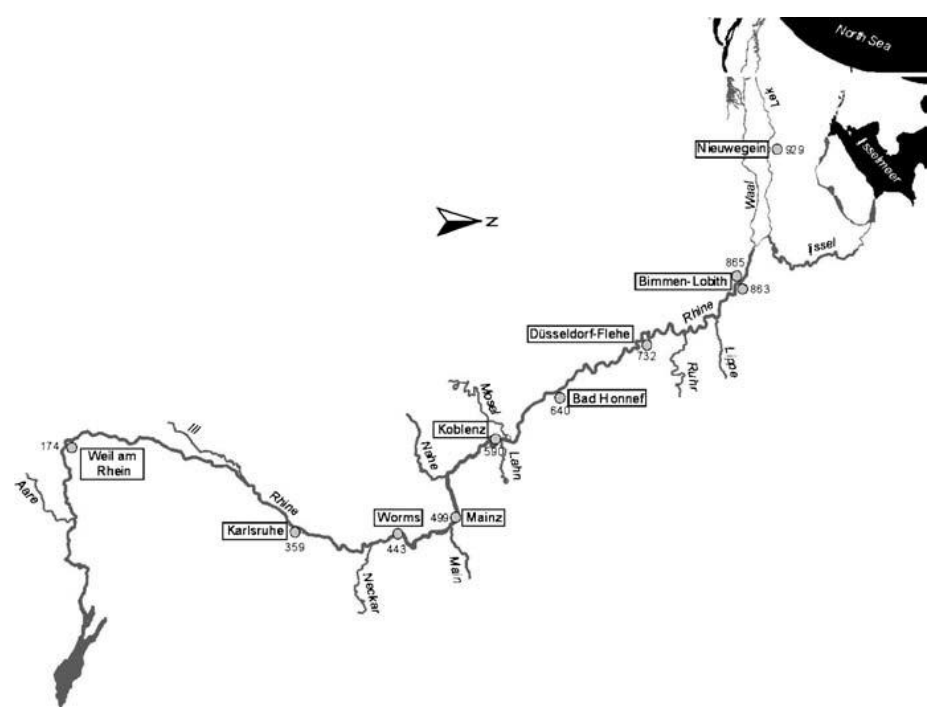

\footnotetext{
${ }^{8}$ https://www.aprona.net/FR/observatoire-eau/presentation-observatoire-eau.html
} 
551 Ces stations ont une fréquence de mesure très élevée, une fois toutes les 30 minutes, et sont 552 surveillées par des centres d'alarme internationaux 24 heures sur 24 . En cas de pollution accidentelle, 553 le centre d'alarme de la station concernée informe les centres d'alarme à l'aval de cette pollution en 554 donnant des informations sur les substances et la quantité déversées dans l'eau et la durée de 555 l'accident.

556 Les stations d'alerte sont localisées à l'aval de chaque pays et surveillent plus de 300 paramètres 557 concernant les caractéristiques chimiques, mais aussi des caractéristiques physiques et biologiques 558 des eaux du Rhin [DIEHL et al. 2005].

559 Ce réseau a été complété par 47 autres stations de surveillance complémentaire [COMMISSION 560 INTERNATIONALE POUR LA PROTECTION DU RHIN, 2016]. Ces stations s'étendent tout au long 561 du Rhin des Alpes à la mer du Nord. La Commission Internationale pour la Protection du Rhin a fait en 562 sorte que les méthodes de mesure soient homogènes d'un pays à l'autre.

564 Après l'accident de Sandoz en 1988, l'Agence de l'eau Rhin-Meuse et la région Alsace ont demandé 565 la mise en place d'une station d'alerte en amont du canal de Huningue dans le Haut-Rhin (Figure 16).

566 [ALTMEYER, 2013]. Cette station a pour mission de contrôler la qualité des eaux en amont de la prise 567 d'eau du canal de Huningue pour éviter à toute eau polluée d'atteindre la nappe phréatique d'Alsace 568 [APRONA, 2012]. La station est financée par la région Grand-Est et l'Agence de l'eau Rhin-Meuse et 569 gérée par l'APRONA (depuis 1997). La qualité est mesurée une fois toutes les 30 minutes. 10 alertes 570 ont lieu en moyenne chaque année. En cas de danger, le préfet, sur proposition de la DREAL Grand571 Est, demande de fermer le canal de Huningue pour protéger la ressource en eau à l'aval. En 572 moyenne, cette décision est prise 3 fois par an [GRAVELINE et al., 2006]. 




\subsubsection{Le Réseau d'Intérêt Départemental (RID)}

578 Le Conseil Général du Bas-Rhin créé en 1998 le Réseau d'Intérêt Départemental (RID) [CONSEIL 579 DEPARTEMENTAL BAS-RHIN, 2016]. A l'origine, ce réseau a pour mission principale de suivre 580 l'impact des politiques d'assainissement du département dans le cadre de la Directive européenne 581 ERU (Eaux Résiduaires Urbaines) de 1991.

Les premières mesures ont lieu en 2000. Les données produites alimentent la banque nationale des données sur l'eau. Les quatre objectifs affichés par le Département [CONSEIL DEPARTEMENTAL BAS-RHIN, 2016] sont :

i) Obtenir plus d'informations sur la qualité de l'eau pour le département du Bas-Rhin ;

ii) Évaluer les tendances à long terme de la qualité de l'eau ;

iv) Communiquer l'information sur la qualité de l'eau à tous les utilisateurs de l'eau.

Le réseau est composé de 70 stations de surveillance (Figure 17) et mesure l'état physico-chimique, biologique et hydro-morphologique des masses d'eau du Bas-Rhin.

591 La fréquence d'échantillonnage est fixée en fonction des paramètres suivants : 


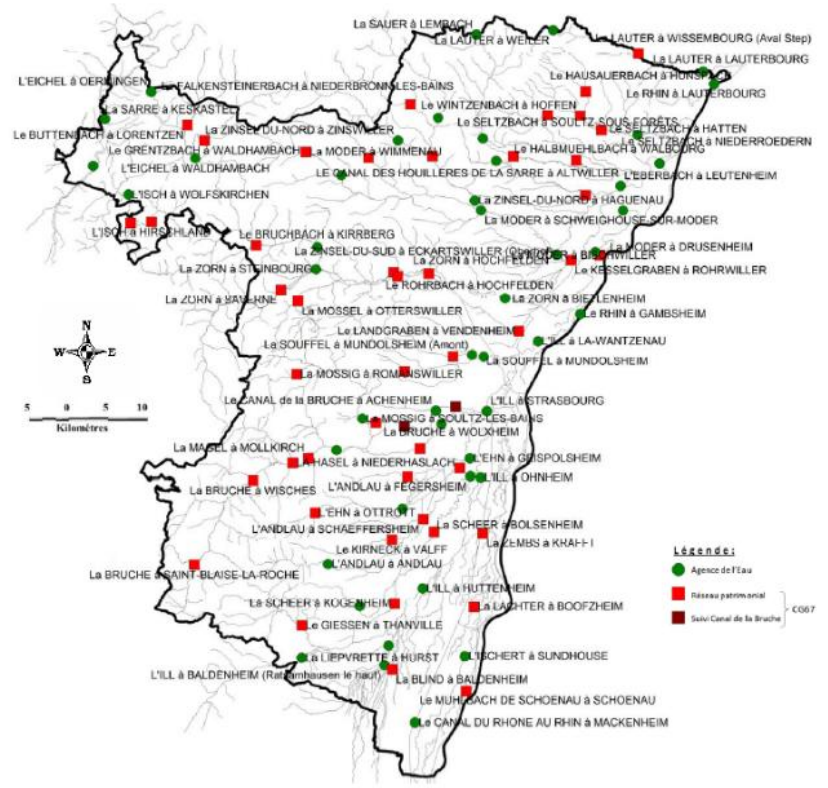

Figure 17 : Réseau d'Intérêt Départemental d'observation de la qualité des cours d'eau du Bas-Rhin [CONSEIL DEPARTEMENTAL BAS-RHIN, 2016]

Le réseau de surveillance RID a été abandonné en 2016. Le Conseil départemental ayant, aujourd'hui, d'autres priorités.

\subsubsection{Le Réseau de suivi des Bassins Miniers}

Depuis la deuxième moitié du $19^{\text {ème }}$ siècle, le nord de la Lorraine a connu une importante activité minière sur le bassin ferrifère [OLLANGIER et HIDALGO, 2011]. L'arrêt progressif de ces activités a eu des conséquences sur le fonctionnement hydrogéologique du bassin tant du point de vue quantitatif que qualitatif. Ainsi, des réseaux de surveillance ont été constitués depuis les années 90 , suite à des arrêtés préfectoraux, pour assurer le suivi de l'ennoyage de ces anciens sites industriels [OLLANGIER et HIDALGO, 2011].

Ce réseau est géré par le BRGM et l'Agence de l'eau Rhin-Meuse. 12 stations surveillent la qualité des eaux souterraines des réservoirs miniers (Figure 18). 


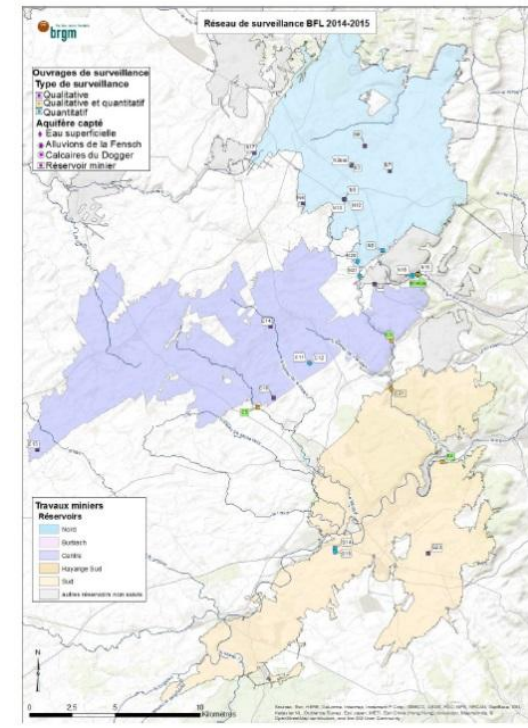

Figure 18: Réseau de surveillance du bassin Ferrifère [GUIGNAT, 2017]

D'autres réseaux après mines existent sur le bassin Rhin-Meuse. Le réseau de contrôle et surveillance de la salinité de la nappe phréatique d'Alsace dans le bassin potassique est apparu en 2007. Ce réseau a pour objectif la collecte d'informations sur la salinité des eaux de surface et souterraine. II est composé, en 2010, de 339 stations de mesure. En 2010, 3000 analyses ont été effectuées dans le bassin potassique [URBAN et BOUCHER, 2011].

\subsubsection{L'Observatoire du cours d'eau des Vosges du Nord}

L'Observatoire des Vosges du Nord est situé dans le parc naturel des Vosges du Nord. Selon la charte du parc, l'objectif est «...de mettre en mouvement le territoire des Vosges du Nord, de rassembler les acteurs et partenaires autour de projets ambitieux et innovants, d'accompagner les élus dans le développement des potentiels de leurs territoires respectifs et surtout de les fédérer derrière un projet commun ».

En 2014, la charte du parc propose la mise en place d'un réseau de surveillance pour mesurer la qualité de l'eau afin de :

- Définir les références en termes de bon état des rivières des Vosges du Nord ;

- Suivre l'évolution de la ressource en eau dans le temps, en particulier après les opérations de restauration; et

- Évaluer l'impact des activités humaines présentes.

La surveillance de l'eau se fait par la mise en place de 31 stations de surveillance : 27 stations de surveillance permanentes et 7 stations de référence [PARC NATIONAL REGIONAL DES VOSGES DU NORD, 2016]. Les paramètres mesurés sont hydro-biologiques, de jaugeage, macro-polluants, micropolluants, matières organiques et hydro-morphologiques. 
Les résultats sont analysés et interprétés par le biais d'une synthèse annuelle qui est produite et diffusée à tous les partenaires dans le cadre d'une réunion de restitution organisée par bassin ou zone géographique.

En France, la surveillance de la qualité de l'eau a débuté avec la loi sur l'eau de 1964. Celle-ci a, par la suite, connu des évolutions au fil des nouvelles lois et Directives européennes sur l'eau. Trois périodes peuvent être ainsi identifiées. La première période, dans les années 1970, reflète le besoin d'avoir un premier éclairage sur la qualité des cours d'eau. Les stations de surveillance sont peu nombreuses et situées uniquement sur les grands cours d'eau à des endroits où une pollution importante est pressentie. La fréquence des mesures est prévue à un rythme de tous les cinq ans. La seconde période, dans les années 1980/1990, marque le désir d'avoir une information plus générale et plus cohérente sur le territoire français, et se manifeste par une multiplication des points de mesure. L'objectif s'élargit également avec la volonté d'identifier les sources de pollution, de suivre l'impact des politiques mises en place ou de cibler des problématiques précises pour répondre aux Directives européennes telles que la Directive ERU ou la Directive nitrate. La dernière période, depuis les années 2000, fait suite à la volonté de la Directive-cadre européenne sur l'eau d'homogénéiser la surveillance sur l'ensemble de l'Europe avec des objectifs clairement identifiés : observation des tendances sur le long terme, identification des sources de pollution et suivi rapproché des masses d'eau en mauvais état,... A ces réseaux nationaux s'ajoutent des réseaux locaux pour des problématiques elles-mêmes locales : risque de pollution accidentelle dans le Rhin, vulnérabilité de la nappe d'Alsace, suivie de pollution d'anciens sites industriels, ...

660 La gestion de ces réseaux de surveillance ; à savoir : choix des paramètres à mesurer, nombre et 661 localisation des stations de surveillance, fréquence de la mesure,....; se fait aux différentes échelles 662 géographiques : au niveau européen avec les circulaires DCE, au niveau national avec des décrets ou 663 arrêtés ministériels, au niveau des bassins avec les Agences de l'eau, les Comités de bassin et les 664 préfets de bassin, mais également par des acteurs comme l'AFB, le BRGM, la DREAL, l'ARS, les 665 Départements ou des associations comme l'APRONA.

666 Les différents réseaux présentés peuvent être rangés dans trois grandes catégories : les réseaux de 667 Long terme (Réseau INP, Réseau patrimonial du RNB, Réseau Nitrate, Réseau de surveillance des 668 pesticides, Réseau du contrôle de surveillance de la DCE, RESALTT, Réseau de surveillance de la 669 qualité de la nappe d'Alsace,...) dont l'objectif est de suivre les tendances dans le temps. Les stations 670 sont ainsi placées de façon régulière et la fréquence de la mesure peut être très espacée, jusqu'à une 671 mesure tous les 5 ou 6 ans; les réseaux de Court terme (Réseau de surveillance continue du Rhin, 
Réseaux de surveillance sanitaire de l'ARS,...) dont l'objectif est de réagir rapidement à une dégradation de la qualité pouvant générer des dommages immédiats. Les stations sont ainsi situées dans les endroits à risque et la fréquence de la mesure peut être quasiment en continu. Enfin des réseaux que l'on pourra qualifier de Moyen terme (Réseaux d'utilisation et d'impact du RNB, Réseaux de contrôle d'enquête et additionnel de la DCE, Réseau de captage prioritaire, RID,...) avec des objectifs opérationnels clairement identifiés comme l'identification des sources de pollution, le suivi de l'impact des politiques mises en œuvre. La fréquence de la mesure est la plupart du temps pluriannuelle.

Comme nous l'avons ainsi vu dans cet article, de très nombreux réseaux de surveillance se superposent sur le territoire français, générant d'innombrables données. Parallèlement, la tendance actuelle est à une limitation des moyens pour effectuer cette surveillance, nouvelles compétences pour les Départementaux, réduction des budgets des Agences de l'eau,.... Se pose ainsi la question de la rationalité de ces réseaux. Comment les données influencent-elles la décision publique pour améliorer la qualité des eaux ? Quel est l'impact de la localisation et de la fréquence de la mesure sur la pertinence de ces décisions ? Ces questions de recherche méritent d'être approfondies pour parvenir à une optimisation de ces réseaux.

Remerciement : Nous tenons à remercier chaleureusement Corinne Grac (ENGEES), Guillaume Demortier (Agence de l'eau Rhin-Meuse) et Pierre-Louis Tisserant (DREAL Grand-Est), pour le temps qu'ils nous ont consacré, et, sans qui, ce travail n'aurait pas été possible.

\section{Bibliographie}

AGENCE DE L'EAU RHIN MEUSE (2016ª) : Résumé du programme de surveillance du district Rhin. Document SDAGE (13).

AGENCE DE L'EAU RHIN MEUSE $\left(2016^{b}\right)$ : Résumé du programme de surveillance du district Meuse. Document SDAGE (14).

AGENCE REGIONALE DE SANTE (2012) : La qualité de l'eau du robinet en France. Synthèse. AGENCE REGIONALE DE SANTE (2018) : La qualité de l'eau du robinet en France. Synthèse, août. ALTMEYER S. (2013) : Intérêts technico-économique de l'analyse en continu de la qualité de l'eau et des milieux au regard des pratiques actuelles. Rapport HYDREOS et ONEMA, juillet. APRONA (2012) : Station d'alerte de Huningue. Rapport APRONA, mai. APRONA (2016) : Rapport de synthèse projet ERMES-Rhin. Rapport. 
BLUM A., LOPEZ B., GUILBERT M., NOEL Y., CHERY L. (2010) : Bilan national des efforts de surveillance des eaux souterraines à partir d'ADES. Indicateurs d'évaluation de l'état qualitatif et quantitatif des eaux souterraines : exemples de réalisations et recommandations. Rapport ONEMABRGM, avril.

BLUM A., WINCKEL A., LAURENT A., MICHON J. (2013) : Les efforts de surveillance des eaux souterraines. Rapport, avril.

BOULEAU G. (2014) : "The Co-production of Science and Waterscapes: The case of the Seine and the Rhône Rivers, France. » Geoforum ; 57: 428-257. Hydrology for the Water Management of Large River Basins; 201.

Bulletin Officiel N²002-6 : Annonce $N^{\circ} 14$, Circulaire du 26 Mars 2002 relative au Système national d'information sur l'eau.

CHERY L., MARTIN A., LEGRAND H. (2008) : « Les systèmes de diffusion nationaux de données: exemples du portail ADES. » Techniques Science Méthodes; 2.

720 Circulaire du 13 Juillet 2006 relative à la constitution et la mise en œuvre du programme de surveillance pour les eaux douces de surface en application de la directive 2000/60/CE du 23 Octobre 2000 du Parlement et du conseil établissant un cadre pour une politique communautaire dans le domaine de l'eau.

Circulaire DCE 2007/22 du 11 avril 2007 relative au protocole de prélèvement et de traitement des échantillons des invertébrés pour la mise en œuvre de programme de surveillance sur cours d'eau. COMMISSION EUROPEENNE (2000) : Directive 2000/60/EC of the European Parliament and of the Council of 23 october 2000. Official Journal of the European Communities, L 327/1. CONSEIL DEPARTEMENTAL DU BAS-RHIN (2014) : Bilan de la Campagne 2014, Qualité des Rivières du Bas-Rhin. Rapport.

CONSEIL DEPARTEMENTAL DU BAS-RHIN (2016) : La suivie de la qualité des rivières. Rapport. COMMISSION INTERNATIONALE POUR LA PROTECTION DU RHIN (2016) : International and national water protection along the Rhine, 30 years after Sandoz, where are we today and what remains to be done? Communiqué de presse.

DIEHL P., GERKE T., JEUKEN A., LOWIS J., STEEN R., VAN STEENWIJK J., STOKS P., WILLEMSEN H.-G. (2005) : «Early Warning Strategies and Practices Along the River Rhine. » Handbook of Environmental Chemistry; 5. DOI 10.1007/015. ERMES (2016) : Etat de la nappe phréatique d'Alsace et des aquifères du Sundgau. Premiers résultats sur les nitrates et les pesticides. Rapport.

739 GIGER W. (2009) : " The Rhine red, the fish dead - the 1986 Schweizerhalle disaster, a retrospect 740 and long-term impact assessment. »Environmental Science and Technology in Romania ; 16 : 98 741111. 
GRAVELINE N., MATON L., ELSASS P., RINAUDO J.D. (2006) : Impact of the use of water quality

743 alternative monitoring methods and tools on decision making. Rapport BRGM, décembre.

744 GUIGNAT S. (2017) : Surveillance 2016 des eaux souterraines du basin ferrifère lorrain. Rapport 745 BRGM, octobre.

746 LALLOUETTE V., PETIT K. (2012) : Bilan de la mise en œuvre de la Directive nitrate 2008-2011.

747 Rapport Ministère de l'Ecologie du Développement Durable et de l'Energie.

748 LANZ K., SCHEUER S. (2001) : EEB Handbook on EU Water Policy under the Water Framework

749 Directive. European Environmental Bureau.

750 LARONDE S., PETIT K. (2010) : Bilan national des efforts de surveillance de la qualité des cours

751 d'eau. Rapport ONEMA-EauFrance, avril.

752 LESAGE M. (2013) : Rapport d'évaluation de la politique de l'eau en France. Mission d'évaluation de 753 la politique de l'eau. www.ladocumentationfrancaise.fr.

754 MINISTERE DE LA TRANSITION ECOLOGIQUE ET SOLIDAIRE (2018) : Note technique portant sur 755 la réalisation de la 7ème campagne de surveillance nitrate 2018-2019 au titre de la Directive 756 91/676/CEE, dite nitrate.

757 OLLANGIER S., HIDALGO J. (2011) : Surveillance des eaux souterraines du bassin ferrifère lorrain 758 sur la période 2008-2010. Rapport BRGM, décembre.

759 PARC NATUREL REGIONAL DES VOSGES DU NORD (2016) : Vosges du Nord. Bilan d'activités.

760 PETIT K. (2010) : Les efforts de surveillance de la qualité des cours d'eau. Rapport EauFrance, juin.

761 ROUX J.-C. (sous la dir. de) (2006) : Aquifère et eaux souterraines en France. Ouvrage collectif. 762 Collection Scientifique et Technique. BRGM éditions. 2 tomes.

763 URBAN S., BOUCHER J. (2011) : Contrôle et surveillance de la salinité de la nappe phréatique 764 d'Alsace dans le bassin potassique et à son aval (68). Rapport de synthèse BRGM, juillet.

765 VAN DIJK G.M., MARTEIJNE E., LEIDIG A. (1995) : "Ecological rehabilitation of the river Rhine: 766 plans, progress, and perspectives. » Regulated Rivers Research and Management; 11: 377-388. 